\title{
When do we need more than local compression to control intraoral haemorrhage?
}

\author{
Jun-Bae Sohn', Ho Lee', Yoon-Sic Han', Da-Un Jung², Hye-Young Sim², Hee-Sun Kim², Sohee Oh \\ ${ }^{1}$ Department of Oral and Maxillofacial Surgery, Seoul Metropolitan Government-Seoul National University (SMG-SNU) \\ Boramae Medical Center, Seoul, \\ ${ }^{2}$ Section of Dentistry, SMG-SNU Boramae Medical Center, Seoul, \\ ${ }^{3}$ Medical Research Collaborating Center, SMG-SNU Boramae Medical Center, Seoul, Korea
}

\begin{abstract}
J Korean Assoc Oral Maxillofac Surg 2019;45:343-350)
Objectives: The aims of this study were to determine the effectiveness of local compression in patients presenting to the emergency room with intraoral bleeding and to identify when complex haemostatic measures may be required.

Materials and Methods: Five hundred forty patients who had experienced intraoral haemorrhage were retrospectively reviewed. The outcome variable was the haemostasis method used, i.e., simple (local compression with gauze) or complex (an alternative method after local compression has failed). Predictor variables were sex, age, American Society of Anesthesiologists (ASA) class, hepatic cirrhosis, bleeding disorder, use of antithrombotic agents, and site/cause of haemorrhage.

Results: The mean patient age was $48.9 \pm 23.9$ years, $53.5 \%$ were male, $42.8 \%$ were ASA class II or higher, and $23.7 \%$ were taking antithrombotic agents. Local compression was used most often (68.1\%), followed by local haemostatic agents, sutures, systemic tranexamic acid or blood products, and electrocautery. The most common site of bleeding was the gingiva (91.7\%), and the most common cause was tooth extraction (45.7\%). Risk factors for needing a complex haemostasis method were use of antithrombotic agents (odds ratio 2.047, $P=0.009$ ) and minor oral surgery (excluding extraction and implant procedures; odds ratio $6.081, P=0.001$ ).

Conclusion: A haemostasis method other than local compression may be needed in patients taking antithrombotic agents or having undergone minor oral surgery.
\end{abstract}

Key words: Haemorrhage, Emergency treatment, Haemostasis, Anticoagulants, Oral surgery

[paper submitted 2019. 8. 17 / revised 2019. 10. 5 / accepted 2019. 10. 28]

\section{Introduction}

Intraoral haemorrhage as a result of a dental procedure or trauma may cause life-threatening complications, such as airway obstruction or hypovolemic shock ${ }^{1-3}$. Therefore, rapid haemostasis may be needed. The most common haemostasis method used for intraoral bleeding is local compression. If

\section{Ho Lee}

Department of Oral and Maxillofacial Surgery, Seoul Metropolitan Government-Seoul National University Boramae Medical Center, 20 Boramae-ro 5-gil, Dongjak-gu, Seoul 07061, Korea

TEL: +82-2-870-2496 FAX: +82-2-831-0714

E-mail:neo0224@gmail.com

ORCID: https://orcid.org/0000-0002-0413-2954

(c) This is an open-access article distributed under the terms of the Creative Commons Attribution Non-Commercial License (http://creativecommons.org/ licenses/by-nc/4.0/), which permits unrestricted non-commercial use, distribution, and reproduction in any medium, provided the original work is properly cited. Copyright (C) 2019 The Korean Association of Oral and Maxillofacial Surgeons. All rights reserved. this method fails, additional local haemostatic measures, such as suturing, can be applied. Aggressive systemic intervention, i.e., transfusion of blood products, ligation of the causative vessel(s), or embolisation, may also be necessary ${ }^{4,5}$.

Systemic factors that can increase the risk of intraoral haemorrhage include antithrombotic agents, hepatic cirrhosis, leukaemia, and inherited bleeding disorders. Local risk factors include highly invasive surgery, extensive soft tissue trauma during surgery, and inflammation at the surgical site. The methods used to prevent postoperative haemorrhage are well known ${ }^{6-9}$. However, most studies have focused on preoperative adjustment of doses of antithrombotic agents. Although there have been some systematic reviews of haemostasis in patients with clinically significant bleeding ${ }^{10}$, such as delayed or secondary haemorrhage, these reviews only mentioned that minor bleeding events were well controlled by local haemostatic measures, regardless of preoperative ad- 
justment of oral anticoagulant therapy ${ }^{11,12}$. There has been no research on factors associated with an increased need to use more than local compression to achieve haemostasis ${ }^{13,14}$.

The aims of this study were to determine the effectiveness of local compression in patients visiting the emergency room with intraoral haemorrhage and to identify patients in whom a haemostatic method other than local compression may be required.

\section{Materials and Methods}

The medical records of all patients who visited our institution with intraoral haemorrhage were retrospectively reviewed during the period from January to June 2019. The inclusion criterion was admission to the Emergency Department either with gingival haemorrhage (K0688) or oral haemorrhage (K137) according to the International Statistical Classification of Diseases and Related Health Problems 10th revision (ICD-10) between 2010 and 2018. The exclusion criteria were intraoral haemorrhage originating from a site other than the oral cavity proper and uncertainty regarding antithrombotic treatment. Two oral and maxillofacial surgeons (J.B.S. and Y.S.H.), each with at least 3 years of experience, evaluated the clinical parameters; if necessary, consensus was reached by discussion. All data collected were checked for missing values and outliers.

The study protocol was approved by the Institutional Review Board of Seoul Metropolitan Government-Seoul National University Boramae Medical Center (approval No. 102019-23) and conducted according to the tenets of the 1964 Declaration of Helsinki and its later amendments. The need for informed consent was waived in view of the retrospective nature of the study and the anonymity of the data.

\section{Haemostasis protocol and clinical setting}

The need for haemostasis in a patient who arrives with intraoral haemorrhage in our Emergency Department is determined by an emergency physician with more than 2 years of experience. If haemostasis is deemed unnecessary, medical support is provided and the patient is discharged after an hour of observation. If haemostasis is necessary, local compression using a small amount of sterile gauze is applied to the site of the haemorrhage for an hour. Blood tests are only performed when deemed necessary by the emergency physician. An oral and maxillofacial surgeon with at least 2 years of clinical experience is called to perform haemostasis if local compres- sion fails. Depending on the cause and location of the haemorrhage, control measures may include application of a local haemostatic agent, sutures, and bipolar or monopolar electrocautery. If the haemorrhage is associated with a mobilised tooth affected by chronic periodontitis, the causative tooth is extracted and a local haemostatic measure is applied. Local haemostatic agents used during the study period included a gelatine sponge (Cutanplast Dental; Curesys, Seoul, Korea), an absorbent collagen dressing (Ateloplug; TRM Korea, Seoul, Korea), and oxidised regenerated cellulose (Surgicel Original; Ethicon, Somerville, NJ, USA). Suturing was performed using non-absorbable uncoated polyamide (Dafilon 4/0; B Braun Surgical SA, Barcelona, Spain). If necessary, these local methods were supplemented by a 500-mg intravenous injection of tranexamic acid (Shinpoong Pharmaceutical, Seoul, Korea) or transfusion of fresh frozen plasma or packed red blood cells. After confirmation of haemostasis, the patient was discharged after an hour of close observation.

\section{Study variables}

The study outcome was whether or not a simple or complex haemostatic method was needed to achieve haemostasis. The haemostasis method was defined as simple if no treatment or only local compression was required or complex if application of a local haemostatic agent, sutures, electrocautery, extraction of a causative tooth, or intravenous administration of tranexamic acid or blood products was needed. Local compression was defined as simple direct local compression only with a small piece of sterile gauze applied to the site of haemorrhage. When more than one method was used for local haemostasis, the final method used was recorded. The intervention was recorded as systemic if intravenous blood products were administered regardless of whether or not a local haemostasis method was used. Potential predictive variables included sex, age, American Society of Anesthesiologists (ASA) class, hepatic cirrhosis, bleeding disorder, history of antithrombotic treatment, and site and cause of haemorrhage.

\section{Statistical analysis}

Continuous variables were analysed using the Student's ttest or Mann-Whitney U test according to the KolmogorovSmirnov test for normality assumption, and categorical variables were analysed using the Pearson's chi-square test or Fisher's exact test. Multivariable logistic regression with 
backward elimination was performed for variables that were found to be statistically significant in univariable logistic regression analyses. The final model was selected based on the Akaike information criterion. The Hosmer-Lemeshow test was performed to assess goodness-of-fit for the final fitted model. All statistical analyses were performed using IBM SPSS Statistics for Windows (ver. 20.0; IBM, Armonk, NY, USA). A $P$-value $<0.05$ was considered statistically significant.

\section{Results}

\section{Patient characteristics in the simple and complex haemostasis groups}

Six hundred eight patients who met the inclusion criteria were screened against the exclusion criteria, after which 540 were eligible for inclusion in the study. Eighty-four patients required a complex haemostasis method. The most common method used was local compression (68.1\%), followed by local haemostatic agents, sutures, systemic blood products, bipolar or monopolar electrocautery, and extraction of a causative tooth. Eighty-eight patients did not require treatment for haemostasis.(Fig. 1) Excluding patients in whom no treatment was administered, local compression alone was adequate for haemostasis in $81.4 \%$ of cases. Intravenous tranexamic acid was administered in five cases and packed red blood cells or fresh frozen plasma in four. Two of these four patients were receiving warfarin, one at a dose of $6.5 \mathrm{mg}$ once daily and the other at a dose of $4.0 \mathrm{mg}$ once daily. The platelet counts in these patients were 221,000 and $157,000 / \mu \mathrm{L}$, respectively, with respective prothrombin time-international normalised ratio (PT-INR) values of 4.38 and 6.60 . The remaining two patients were not taking antithrombotic agents; one had idiopathic thrombocytopenic purpura (platelet count, 50,000; PTINR, 1.01) and the other had alcoholic liver cirrhosis (platelet count, 77,000; PT-INR, 1.27). No invasive procedures, such as embolisation of the affected blood vessels, were required in the emergency room.

The general characteristics of the simple and complex haemostasis groups are summarised in Table 1. The mean age was 48.9 years (range, 1-93 years), and 53.5\% were male. ASA class II or higher systemic disease was present in 231 cases, hepatic cirrhosis in 17 cases, and a bleeding disorder (thrombocytopenic purpura) in 2 cases. One hundred and twenty-eight patients were receiving antithrombotic agents. The antiplatelet agents used were aspirin, clopidogrel bisulphate, cilostazol, and triflusal; and the anticoagulants used were warfarin, apixaban, and dabigatran.(Supplementary Table S1) Whether or not the antithrombotic agents were

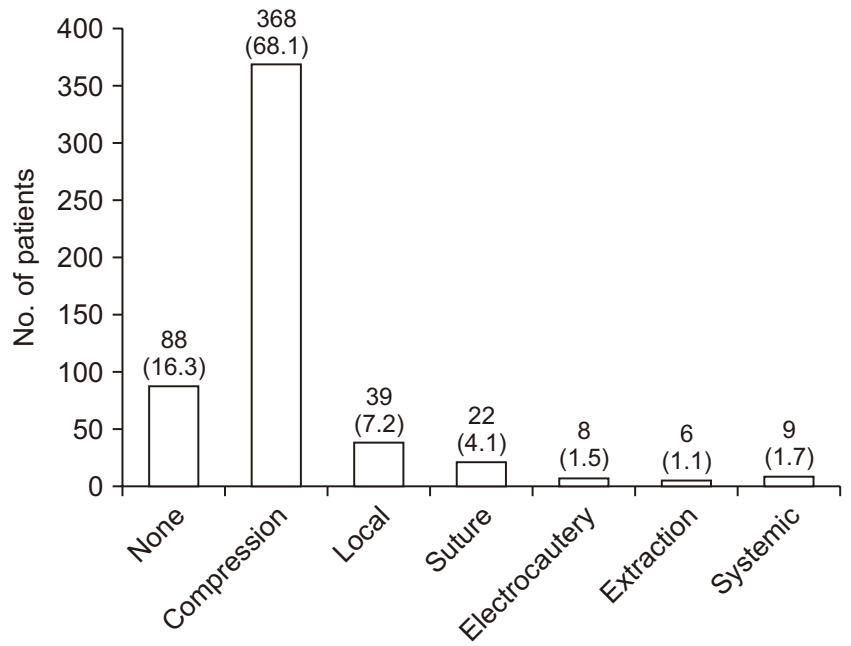

Fig. 1. Method of haemostasis used for intraoral haemorrhage. The values are presented as the number (\%). (None: no treatment, Compression: simple direct local compression only with a small piece of sterile gauze applied to the site of haemorrhage, Local: local haemostatic agent, Electrocautery: bipolar or monopolar electrocautery, Extraction: extraction of causative tooth, Systemic: systemic administration of tranexamic acid or blood products) Jun-Bae Sohn et al: When do we need more than local compression to control intraoral haemorrhage?. J Korean Assoc Oral Maxillofac Surg 2019

Table 1. Description of risk factors according to whether a simple or complex method of haemostasis was used for intraoral haemorrhage

\begin{tabular}{lcccc}
\hline \multicolumn{1}{c}{ Risk factor } & Total $(\mathrm{n}=540)$ & Simple $(\mathrm{n}=456)$ & Complex $(\mathrm{n}=84)$ & $P$-value \\
\hline Age (yr) & $48.9 \pm 23.9$ & $48.0 \pm 24.4$ & $53.7 \pm 20.1$ & $0.085^{1}$ \\
Sex (male) & $289(53.5)$ & $246(53.9)$ & $43(51.2)$ & $0.642^{2}$ \\
ASA II or higher & $231(42.8)$ & $186(40.8)$ & $45(53.6)$ & $0.030^{2 *}$ \\
Hepatic cirrhosis & $17(3.1)$ & $13(2.9)$ & $4(4.8)$ & $0.318^{3}$ \\
Bleeding disorder & $2(0.4)$ & $1(0.2)$ & $1(1.2)$ & $0.287^{3}$ \\
Use of an antithrombotic agent & $128(23.7)$ & $99(21.7)$ & $29(34.5)$ & $0.011^{2 *}$ \\
\hline
\end{tabular}

(Simple: simple method of haemostasis, Complex: complex method of haemostasis, ASA: American Society of Anesthesiologists)

${ }^{1}$ Mann-Whitney U test. ${ }^{2}$ Chi-square test. ${ }^{3}$ Fisher's exact test.

$* P<0.05$.

Values are presented as mean \pm standard deviation or number $(\%)$.

Jun-Bae Sohn et al: When do we need more than local compression to control intraoral haemorrhage?. J Korean Assoc Oral Maxillofac Surg 2019 
discontinued before the dental procedures were performed is unclear; however, all patients on antithrombotic agents were taking these medications at the time of their emergency room visit.

The most common site of haemorrhage was the gingiva (91.7\%), followed by the buccal mucosa, tongue, palatal mucosa, and lip.(Fig. 2) The simple and complex haemostasis groups are compared according to the cause of haemorrhage in Fig. 3. The most common cause of haemorrhage was tooth extraction (45.7\%), followed by installation of a dental implant fixture, trauma, local inflammation caused by peri- odontitis, basic periodontal treatment, minor oral surgery (excluding tooth extraction and installation of a dental implant), intraoral insertion of a Penrose drain, and cancer. The specific types of minor oral surgery performed are described in Table 2. In a further 3 cases, spontaneous bleeding was caused by idiopathic thrombocytopenic purpura, mucosal abrasion by severe xerostomia, and eruption of a permanent tooth, respectively.
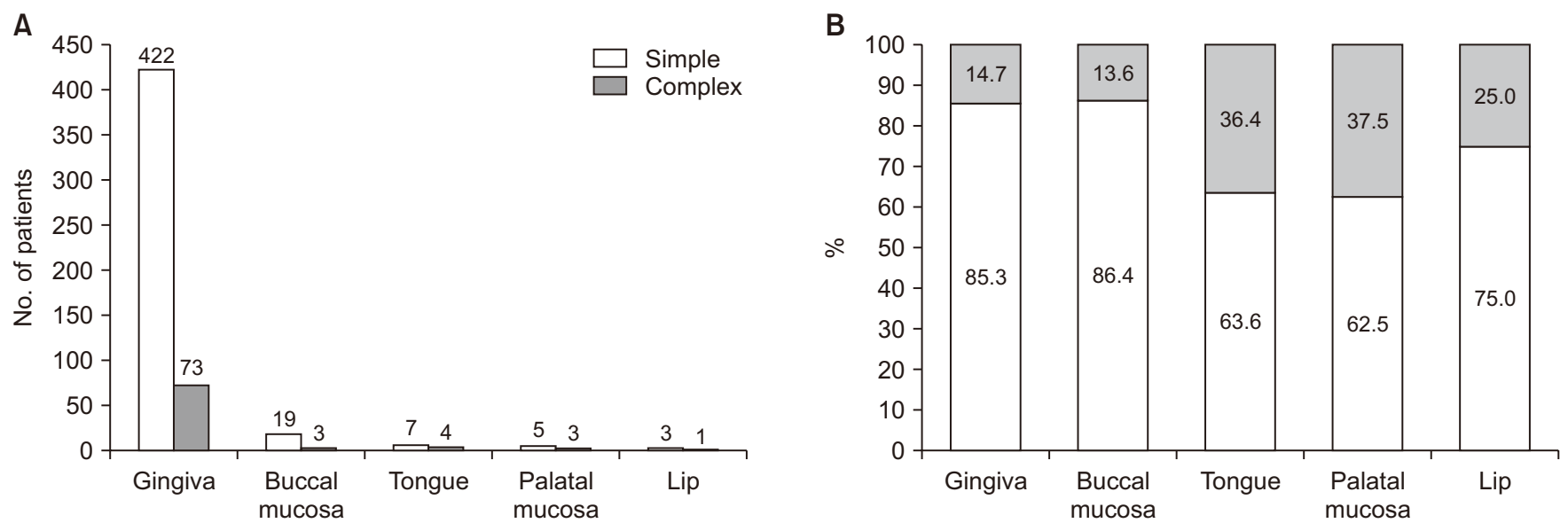

Fig. 2. A. Number of patients. B. Percentile of patients. Comparison of cases stratified by whether or not a simple or complex method of haemostasis was performed according to the site of haemorrhage. $P=0.169$, univariable logistic regression test. The values are presented as the number or percentile. (Simple: simple method of haemostasis, Complex: complex method of haemostasis) Jun-Bae Sohn et al: When do we need more than local compression to control intraoral haemorrhage?. J Korean Assoc Oral Maxillofac Surg 2019
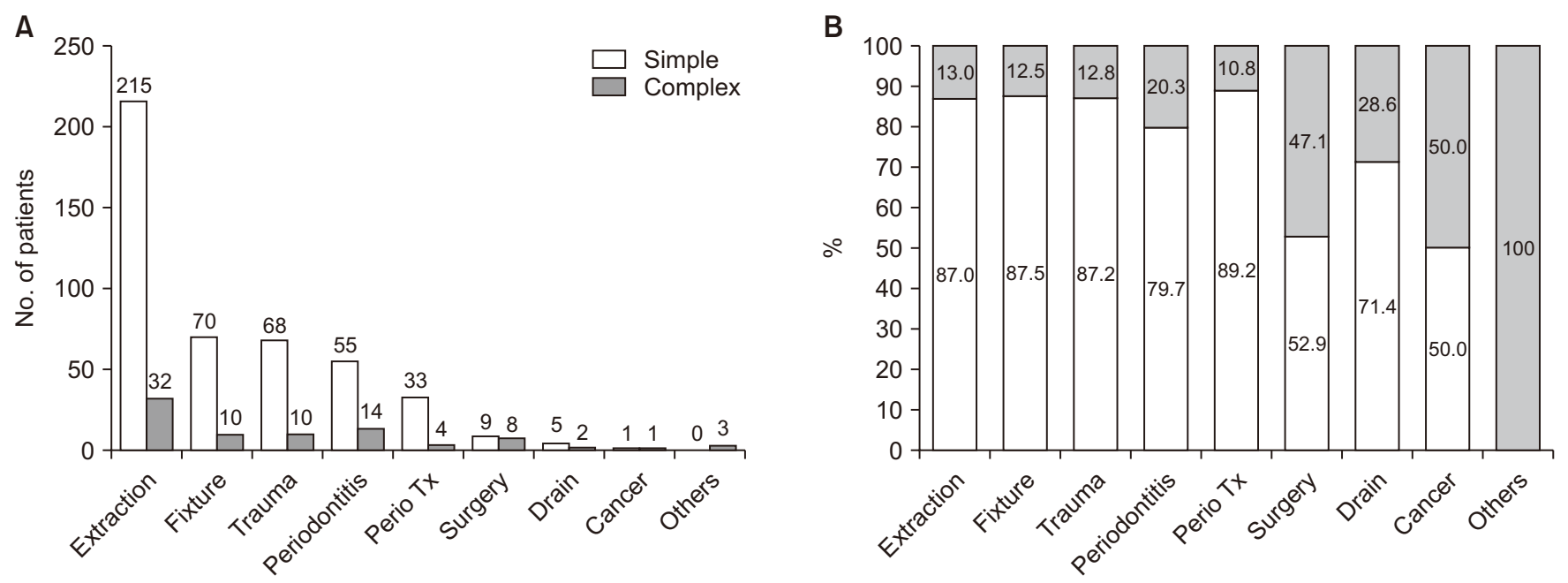

Fig. 3. A. Number of patients. B. Percentile of patients. Comparison of cases stratified by whether or not a simple or complex method of haemostasis was performed according to the cause of haemorrhage. $P=0.019$, univariable logistic regression test. Values are presented as the number or percentile. (Simple: simple method of haemostasis, Complex: complex method of haemostasis, Extraction: tooth extraction, Fixture: installation of a dental implant fixture, Trauma: physical trauma to tooth or gingiva, Periodontitis: local inflammation caused by periodontitis, Perio Tx: basic periodontal treatment including scaling or subgingival curettage, Surgery: minor oral surgery excluding tooth extraction and dental implant surgery, Drain: intraoral Penrose drain insertion, Cancer: oral cancer)

Jun-Bae Sohn et al: When do we need more than local compression to control intraoral haemorrhage?. J Korean Assoc Oral Maxillofac Surg 2019 


\section{Factors affecting the haemostasis method used}

Univariable logistic regression analysis revealed significant associations of use of a complex haemostasis method with older age, ASA class II or higher systemic disease, and use of an antithrombotic agent. A complex haemostasis method was used more often after minor oral surgery than after tooth extraction.(Table 3) Multivariable logistic regression analysis revealed significant associations between the need for a complex haemostasis method and use of an antithrombotic agent and minor oral surgery.(Table 4)

\section{Discussion}

The most straightforward haemostatic method used for intraoral bleeding is local compression. However, a more

Table 2. Types of minor oral surgery and number of patients

\begin{tabular}{lc}
\hline \multicolumn{1}{c}{ Minor oral surgery } & No. of patients \\
\hline Total & 17 \\
Free gingival graft & 7 \\
Bone graft for alveolar atrophy & 3 \\
Periodontal flap surgery & 3 \\
Vestibuloplasty & 1 \\
Orthodontic microscrew & 1 \\
Plate removal & 1 \\
Incision and drainage without drain & 1 \\
\hline
\end{tabular}

Jun-Bae Sohn et al: When do we need more than local compression to control intraoral haemorrhage?. J Korean Assoc Oral Maxillofac Surg 2019 complex method is needed if this is ineffective, which entails additional cost and time consumption. Complex methods include local haemostatic agents, suturing, electrocautery, extraction of the causative tooth, and systemic administration of tranexamic acid or blood products. Local haemostatic methods include application of a gelatine sponge, absorbent collagen dressing, or oxidised regenerated cellulose. This study aimed to determine the effectiveness of local compression in patients presenting to the emergency room with intraoral bleeding and to assess the risk factors prompting a more complex approach. Local compression was usually sufficient for haemostasis. Use of a complex haemostatic approach was associated with use of an antithrombotic agent and minor oral surgery, such as a free gingival graft, alveolar bone graft, or periodontal flap surgery.

Various local haemostatic methods have been used in dentistry. Most cases of intraoral haemorrhage can be controlled successfully by local haemostatic methods with or without suturing ${ }^{4,15,16}$. In the present study, 39 of 84 patients requiring a complex haemostasis method were successfully treated with local haemostatic agents. Other cases were controlled with alternative local measures, including sutures, electrocautery, and extraction of the causative teeth. However, in 9 of the 540 cases in this study, local haemostasis methods failed and intravenous administration of tranexamic acid or blood products was required. Four patients who required transfu-

Table 3. Results of univariable logistic regression analysis of factors associated with the need for a complex method of haemostasis for intraoral haemorrhage

\begin{tabular}{|c|c|c|c|}
\hline Risk factor & OR & $95 \% \mathrm{CI}$ & $P$-value \\
\hline Age & 1.010 & $1.000-1.021$ & $0.047 *$ \\
\hline Sex (male) & 1.117 & $0.701-1.780$ & 0.642 \\
\hline ASA II or higher & 1.675 & $1.049-2.674$ & $0.031 *$ \\
\hline Hepatic cirrhosis & 1.704 & $0.542-5.358$ & 0.362 \\
\hline Bleeding disorder & 5.482 & $0.340-88.510$ & 0.231 \\
\hline Antithrombotic agent & 1.901 & $1.151-3.141$ & $0.012 *$ \\
\hline Site of haemorrhage ${ }^{1}$ & & & 0.169 \\
\hline Buccal mucosa & 0.913 & $0.263-3.163$ & 0.886 \\
\hline Tongue & 3.303 & $0.943-11.568$ & 0.062 \\
\hline Palatal mucosa & 3.468 & $0.811-14.827$ & 0.093 \\
\hline Lip & 1.927 & $0.198-18.778$ & 0.572 \\
\hline Cause of haemorrhage $^{2}$ & & & $0.032 *$ \\
\hline Installation of a dental implant fixture & 0.960 & $0.449-2.051$ & 0.916 \\
\hline Trauma & 0.988 & $0.462-2.114$ & 0.975 \\
\hline Local inflammation caused by periodontitis & 1.710 & $0.854-3.425$ & 0.130 \\
\hline Basic periodontal treatment & 0.814 & $0.271-2.452$ & 0.715 \\
\hline Minor oral surgery ${ }^{3}$ & 5.972 & $2.149-16.599$ & $0.001 *$ \\
\hline Intraoral Penrose drain & 2.687 & $0.500-14.439$ & 0.249 \\
\hline Cancer & 6.719 & $0.410-110.110$ & 0.182 \\
\hline Other & 10853971746 & 0.000-infinite & 0.999 \\
\hline
\end{tabular}

(OR: odds ratio, CI: confidence interval, ASA: American Society of Anesthesiologists)

${ }^{1}$ Compared with the gingiva. ${ }^{2}$ Compared with tooth extraction. ${ }^{3}$ Excluding tooth extraction and installation of a dental implant fixture.

$* P<0.05$.

Jun-Bae Sohn et al: When do we need more than local compression to control intraoral haemorrhage?. J Korean Assoc Oral Maxillofac Surg 2019 
Table 4. Results of multivariable logistic regression analysis of factors related with the complex method of haemostasis for intraoral haemorrhage

\begin{tabular}{|c|c|c|c|}
\hline Risk factor & OR & $95 \% \mathrm{CI}$ & $P$-value \\
\hline Antithrombotic agent & 2.047 & $1.201-3.535$ & $0.009 *$ \\
\hline Cause of haemorrhage ${ }^{1}$ & & & $0.024 *$ \\
\hline Installation of a dental implant fixture & 0.856 & $0.396-1.850$ & 0.692 \\
\hline Trauma & 0.942 & $0.438-2.028$ & 0.879 \\
\hline Local inflammation caused by periodontitis & 1.420 & $0.694-2.905$ & 0.337 \\
\hline Basic periodontal treatment & 0.668 & $0.218-2.049$ & 0.480 \\
\hline Minor oral surgery $^{2}$ & 6.081 & $2.167-17.067$ & $0.001 *$ \\
\hline Intraoral Penrose drain & 3.107 & $0.575-16.782$ & 0.188 \\
\hline Cancer & 7.766 & $0.472-127.697$ & 0.151 \\
\hline Other & 10230091867 & 0.000-infinite & 0.999 \\
\hline
\end{tabular}

(OR: odds ratio, CI: confidence interval, ASA: American Society of Anesthesiologists)

${ }^{1}$ Compared with tooth extraction. ${ }^{2}$ Excluding tooth extraction and installation of a dental implant fixture.

$* P<0.05$.

Hosmer-Lemeshow goodness-of-fit test, $P=0.805$. Akaike information criterion, 453.473.

Jun-Bae Sohn et al: When do we need more than local compression to control intraoral haemorrhage?. J Korean Assoc Oral Maxillofac Surg 2019

sion had a PT-INR $\geq 4$ at the time of bleeding or had severe systemic disease. In all 9 cases, use of a systemic haemostasis method was successful and without complications.

In previous studies, use of a local haemostatic agent, such as oxidised regenerated cellulose, was more effective than local pressure alone for controlling bleeding after surgery in the palatal region, such as a free gingiva graft ${ }^{17,18}$. In our study, a complex haemostasis method was more likely if the haemorrhage involved the tongue, palate, or lips than the gingival and buccal mucosa. This finding might reflect local anatomical characteristics, i.e., local compression involving the tongue, palate, and lips is more difficult than that involving the gingiva and buccal mucosa. However, we found no statistically significant difference in choice of haemostatic method according to site of haemorrhage.

Antithrombotic agents have been widely used to prevent and treat cardiovascular disease and stroke ${ }^{19,20}$. Therefore, many studies of the complications associated with postoperative bleeding have been performed in patients taking antithrombotic agents. Use of acetylsalicylic acid alone does not significantly increase the risk of bleeding after oral surgery $^{10,21}$. However, patients receiving dual antiplatelet therapy or anticoagulants, such as warfarin and direct oral anticoagulants, are at greater risk of bleeding after minor oral surgery; however, a local haemostasis method at the time of the procedure is usually enough to avoid excessive bleeding ${ }^{11,13,22-24}$. Previous studies have focused mainly on whether or not antithrombotic therapy should be stopped before invasive dental treatment, and an antithrombotic agent's effects on the haemostasis method used in cases of secondary haemorrhage are unclear. In the present study, the haemostasis method used was likely to be more complicated in patients with secondary or delayed haemorrhage using antithrombotic agents.

We found that minor oral surgical procedures, including free gingival grafts, bone grafts for alveolar atrophy, and periodontal flap surgery, but not tooth extraction or installation of a dental implant fixture, increased the likelihood of needing complex haemostasis. Postoperative local compression easily controlled bleeding in an extraction socket. An implant fixture itself functioned as a pressure dressing; and primary closure was possible in implant surgery, which is more favourable for haemostasis ${ }^{25}$. Most of the previous studies on procedure-related bleeding and the haemostasis method applied have focused on the antithrombotic agent used. Scully et al. reported that, with the exception of harvesting of an autogenous bone graft, extensive flap, or osteotomy, use of a local haemostatic agent only reduced bleeding without discontinuation of oral anticoagulant therapy after simple extraction or implant placement ${ }^{14,26}$. Febbo et al. ${ }^{27}$ reported that the number of postoperative bleeding complications increased as the number of teeth extracted increased. In contrast, Gualandro et al. ${ }^{28}$ recommended continuation of antiplatelet therapy in patients undergoing multiple tooth extractions, biopsy, flap surgery, gingivectomy, or alveoloplasty. These researchers suggested that the usual dose of warfarin could be continued in patients undergoing extraction of up to 5 teeth or installation of up to 6 implants. Another study found no correlation between the risk of postoperative bleeding and type of dental surgery $^{23}$.

Most studies of oral bleeding have focused on risk factors that affect bleeding after dental procedures in patients taking medications that affect the mechanism of haemostasis ${ }^{15,29}$. However, no previous study has evaluated the factors determining the method required for effective haemostasis in 
patients with intraoral haemorrhage, regardless of antithrombotic therapy or the dental procedure performed. Moreover, complex haemostasis measures are relatively more costly and time-consuming to perform than local compression. Nevertheless, most of the published studies did not separate simple and complex haemostasis methods. The novelty of our study is that we separated these two methods.

This study has several limitations. First, the study had a retrospective design and was based on review of medical records without direct patient observation. Second, we only focused on the causative dental procedure; we did not consider the details of each procedure, extent of damage caused by surgery, the state of inflammation at the surgical site, and time elapsed since the procedure. Third, we considered only the presence or absence of systemic disease without detailed disease classification. Fourth, we did not consider the platelet count or PT-INR at the time of haemorrhage because such tests were only performed in patients with severe bleeding. Therefore, a larger prospective study that considers these factors is needed.

\section{Conclusion}

Conventional local compression is easily performed and useful when a patient visits the emergency room with intraoral bleeding. However, primary use of another method, such as a haemostatic agent, suturing, electrocautery, or removal of the cause of inflammation, might be preferable for rapid haemostasis in patients who have undergone minor oral surgery, such as a free gingival graft, bone graft for alveolar atrophy, or periodontal flap surgery, while taking antithrombotic agents.

\section{ORCID}

Jun-Bae Sohn, https://orcid.org/0000-0002-5134-7085

Ho Lee, https://orcid.org/0000-0002-0413-2954

Yoon-Sic Han, https://orcid.org/0000-0001-8060-5330

Da-Un Jung, https://orcid.org/0000-0002-0278-5476

Hye-Young Sim, https://orcid.org/0000-0002-3338-4245

Hee-Sun Kim, https://orcid.org/0000-0002-1445-6565

Sohee Oh, https://orcid.org/0000-0002-3010-448X

\section{Authors' Contributions}

J.B.S. and Y.S.H. participated in data collection and wrote the manuscript. H.L. participated in the study design and coordination and wrote the manuscript. D.U.J., H.Y.S., and H.S.K. participated in coordination and helped to draft the manuscript. S.O. performed the statistical analysis and interpretation of data. All authors read and approved the final manuscript.

\section{Acknowledgements}

We would like to thank Editage (http://www.editage.com) for editing and reviewing this manuscript for English language.

\section{Ethics Approval and Consent to Participate}

The study protocol was approved by the Institutional Review Board of Seoul Metropolitan Government-Seoul National University Boramae Medical Center (approval No. 102019-23), and the need for informed consent was waived in view of the retrospective nature of the study and the anonymity of the data.

\section{Supplementary Materials}

Supplementary data is available at http://www.jkaoms.org.

\section{Conflict of Interest}

No potential conflict of interest relevant to this article was reported.

\section{References}

1. Ziffer AM, Scopp IW, Beck J, Baum J, Berger AR. Profound bleeding after dental extractions during dicumarol therapy. N Engl J Med 1957;256:351-3.

2. Cannon PD, Dharmar VT. Minor oral surgical procedures in patients on oral anticoagulants--a controlled study. Aust Dent J 2003;48:115-8.

3. Bouloux GF, Perciaccante VJ. Massive hemorrhage during oral and maxillofacial surgery: ligation of the external carotid artery or embolization? J Oral Maxillofac Surg 2009;67:1547-51.

4. Bajkin BV, Popovic SL, Selakovic SD. Randomized, prospective trial comparing bridging therapy using low-molecular-weight heparin with maintenance of oral anticoagulation during extraction of teeth. J Oral Maxillofac Surg 2009;67:990-5.

5. Costa FW, Rodrigues RR, Sousa LH, Carvalho FS, Chaves FN, Fernandes CP, et al. Local hemostatic measures in anticoagulated patients undergoing oral surgery: a systematized literature review. Acta Cir Bras 2013;28:78-83.

6. Scully C, Wolff A. Oral surgery in patients on anticoagulant therapy. Oral Surg Oral Med Oral Pathol Oral Radiol Endod 2002;94:57-64.

7. Medina JB, Andrade NS, de Paula Eduardo F, Bezinelli L, Franco 
JB, Gallottini M, et al. Bleeding during and after dental extractions in patients with liver cirrhosis. Int J Oral Maxillofac Surg 2018;47:1543-9.

8. Rasaratnam L, Chowdary P, Pollard D, Subel B, Harrington C, Darbar UR. Risk-based management of dental procedures in patients with inherited bleeding disorders: Development of a Dental Bleeding Risk Assessment and Treatment Tool (DeBRATT). Haemophilia 2017;23:247-54.

9. Sprenker C, Omar HR, Powless RA, Mangar D, Camporesi E. Massive oral bleeding after full-mouth extraction in a patient with B-cell lymphocytic leukemia/small lymphocytic lymphoma reversed with recombinant activated factor VII. J Am Dent Assoc 2016;147:142-5.

10. Krishnan B, Shenoy NA, Alexander M. Exodontia and antiplatelet therapy. J Oral Maxillofac Surg 2008;66:2063-6.

11. Kämmerer PW, Frerich B, Liese J, Schiegnitz E, Al-Nawas B. Oral surgery during therapy with anticoagulants-a systematic review. Clin Oral Investig 2015;19:171-80.

12. Madrid C, Sanz M. What influence do anticoagulants have on oral implant therapy? A systematic review. Clin Oral Implants Res 2009;20 Suppl 4:96-106.

13. Wahl MJ, Pinto A, Kilham J, Lalla RV. Dental surgery in anticoagulated patients--stop the interruption. Oral Surg Oral Med Oral Pathol Oral Radiol 2015;119:136-57.

14. Eichhorn W, Burkert J, Vorwig O, Blessmann M, Cachovan G, Zeuch J, et al. Bleeding incidence after oral surgery with continued oral anticoagulation. Clin Oral Investig 2012;16:1371-6.

15. Morimoto Y, Nakatani T, Yokoe C, Kudo C, Hanamoto H, Niwa H. Haemostatic management for oral surgery in patients supported with left ventricular assist device--a preliminary retrospective study. Br J Oral Maxillofac Surg 2015;53:991-5.

16. Vezeau PJ. Topical hemostatic agents: what the oral and maxillofacial surgeon needs to know. Oral Maxillofac Surg Clin North Am 2016;28:523-32.

17. Rossmann JA, Rees TD. A comparative evaluation of hemostatic agents in the management of soft tissue graft donor site bleeding. J Periodontol 1999;70:1369-75.

18. Ozcan M, Ucak O, Alkaya B, Keceli S, Seydaoglu G, Haytac MC. Effects of platelet-rich fibrin on palatal wound healing after free gingival graft harvesting: a comparative randomized controlled clinical trial. Int J Periodontics Restorative Dent 2017;37:e270-8.

19. Arora RR, Rai F. Antiplatelet intervention in acute coronary syn- drome. Am J Ther 2009;16:e29-40.

20. Su Q, Li C, Long F, Chen B, Wan Z, Wu Y, et al. Effects of a health promotion program on medication adherence to antiplatelet therapy among ischemic stroke patients in Hainan Province, China. Vascular 2017;25:242-8.

21. Pototski M, Amenábar JM. Dental management of patients receiving anticoagulation or antiplatelet treatment. J Oral Sci 2017;49:253-8.

22. Nathwani S, Martin K. Exodontia in dual antiplatelet therapy: the evidence. Br Dent J 2016;220:235-8.

23. Li L, Zhang W, Yang Y, Zhao L, Zhou X, Zhang J. Dental management of patient with dual antiplatelet therapy: a meta-analysis. Clin Oral Investig 2019;23:1615-23.

24. Napeñas JJ, Oost FC, DeGroot A, Loven B, Hong CH, Brennan MT, et al. Review of postoperative bleeding risk in dental patients on antiplatelet therapy. Oral Surg Oral Med Oral Pathol Oral Radiol 2013;115:491-9.

25. Bacci C, Berengo M, Favero L, Zanon E. Safety of dental implant surgery in patients undergoing anticoagulation therapy: a prospective case-control study. Clin Oral Implants Res 2011;22:151-6.

26. Scully C, Hobkirk J, Dios PD. Dental endosseous implants in the medically compromised patient. J Oral Rehabil 2007;34:590-9.

27. Febbo A, Cheng A, Stein B, Goss A, Sambrook P. Postoperative bleeding following dental extractions in patients anticoagulated with warfarin. J Oral Maxillofac Surg 2016;74:1518-23.

28. Gualandro DM, Yu PC, Calderaro D, Marques AC, Pinho C, Caramelli B, et al. II Guidelines for perioperative evaluation of the Brazilian Society of Cardiology. Arq Bras Cardiol 2011;96(3 Suppl 1):1-68.

29. Morimoto Y, Niwa H, Minematsu K. Risk factors affecting postoperative hemorrhage after tooth extraction in patients receiving oral antithrombotic therapy. J Oral Maxillofac Surg 2011;69:1550-6.

How to cite this article: Sohn JB, Lee H, Han YS, Jung DU, Sim HY, Kim HS, et al. When do we need more than local compression to control intraoral haemorrhage? J Korean Assoc Oral Maxillofac Surg 2019;45:343-350. https://doi.org/10.5125/jkaoms.2019.45.6.343 